\title{
Avaliação Clínico-epidemiológica de Pacientes com Carcinoma de Células Escamosas Oral
}

doi: https://doi.org/10.32635/2176-9745.RBC.2022v68n1.1584

\author{
Epidemiological and Clinical Evaluation of Patients with Oral Squamous Cell Carcinoma \\ Evaluación Epidemiológica y Clínica de Pacientes con Carcinoma Oral de Células Escamosas
}

\begin{abstract}
Júlio César Saraiva Santos'; Carlos Eduardo Moura Carvalho Rochaa; Rafael Everton Assunção Ribeiro da Costa ${ }^{3}$; Eduardo Salmito Soares Pinto4; Ana Luisa Rios Barbosa de Almeida5; João Batista Mendes Teles ${ }^{6}$; Luciana Tolstenko Nogueira”; Lucielma Salmito Soares Pinto ${ }^{8}$
\end{abstract}

\section{RESUMO}

Introduçáo: O câncer bucal corresponde a cerca de 30\% de todos os tumores de cabeça e pescoço. Aproximadamente $90 \%$ dessas neoplasias malignas são carcinomas espinocelulares (CEC) e cerca de 15 mil casos novos são estimados a cada ano no Brasil. Objetivo: Avaliar os aspectos clínico-epidemiológicos de pacientes com CEC oral. Método: Estudo observacional, com delineamento transversal, quantitativo e retrospectivo, a partir da análise de prontuários de pacientes com diagnóstico histopatológico de CEC bucal atendidos em um centro de referência em Oncologia da Região Nordeste do Brasil. Variáveis clínicas e epidemiológicas foram coletadas e analisadas. A análise estatística foi realizada utilizando-se o teste de qui-quadrado $(\mathrm{p} \leq 0,05)$. Resultados: Entre os 298 prontuários avaliados, predominaram pacientes do sexo masculino (75,2\%), com idade média de 60,4 anos, tabagistas e etilistas (62,0\%). A queixa mais frequente foi a presença de ferida ou lesão na boca $(61,1 \%)$, e o local mais comum foi a língua $(62,1 \%)$. As variáveis clínicas revelaram estadiamento clínico avançado (III ou IV) em 76,4\% dos pacientes. Entre os pacientes com idade até 50 anos, houve maior concentração de homens ( $\mathrm{p}=0,015$ ) e maior consumo de álcool do que entre os pacientes acima dos 50 anos $(\mathrm{p}=0,010)$. As demais variáveis não exibiram diferença estatística significante entre os grupos. Conclusáo: As características clínico-epidemiológicas relacionadas ao CEC bucal devem ser consideradas para o planejamento de políticas públicas, a fim de prevenir novos casos e permitir a realização de diagnóstico precoce.

Palavras-chave: carcinoma de células escamosas de cabeça e pescoço/epidemiologia; neoplasias bucais; registros médicos.

\section{ABSTRACT}

Introduction: Oral cancer corresponds to about 30\% of all head and neck tumors. Approximately $90 \%$ of these malignancies are squamous cell carcinomas (SCC) and about 15 thousand new cases are estimated each year in Brazil. Objective: Evaluate the clinical and epidemiological aspects of patients with oral SCC. Method: Observational cross-sectional, quantitative and retrospective design study, based on the analysis of medical records of patients with histopathological diagnosis of oral SCC treated at a reference center in oncology in Brazil's Northeast. Clinical and epidemiological variables were collected and analyzed. Statistical analysis was performed using the chi-square test $(\mathrm{p} \leq 0.05)$. Results: Among the 298 medical records evaluated, male patients predominated $(75.2 \%)$, with an average age of 60.4 years, smokers and alcoholics $(62.0 \%)$. The most reported complaint was the presence of a wound or injury in the mouth $(61.1 \%)$ and the most common location was the tongue $(62.1 \%)$. Clinical variables revealed advanced clinical staging (III or IV) in $76.4 \%$ of patients. Among patients aged up to 50 years, there was high concentration of men $(\mathrm{p}=0.015)$ and greater use of alcohol than among patients over 50 years $(\mathrm{p}=0.010)$. The other variables did not show a statistically significant difference between the groups. Conclusion: The clinical and epidemiological characteristics related to oral SCC should be considered when planning public policies, in order to prevent new cases as well as to allow for early diagnosis.

Key words: squamous cell carcinoma of head and neck/epidemiology; mouth neoplasms; medical records.

\section{RESUMEN}

Introducción: El cáncer oral corresponde a aproximadamente $30 \%$ de todos los tumores de cabeza y cuello. Aproximadamente $90 \%$ de estos tumores malignos son carcinomas de células escamosas (CCE) y se estiman alrededor de 15 mil casos nuevos cada ańo en Brasil. Objetivo: Evaluar los aspectos clínicos y epidemiológicos de los pacientes con CCE oral. Método: Estudio observacional, con diseño transversal, cuantitativo y retrospectivo, basado en el análisis de historias clínicas de pacientes con diagnóstico histopatológico de CCE oral tratados en un centro de referencia en Oncología del Noreste de Brasil. Se recogieron y analizaron variables clínicas y epidemiológicas. El análisis estadístico se realizó mediante la prueba de chi-cuadrado $(\mathrm{p} \leq 0,05)$. Resultados: Entre las 298 historias clínicas evaluadas, predominaron los pacientes del sexo masculino $(75,2 \%)$, con una edad promedio de 60,4 ańos, fumadores y alcohólicos (62,0\%). La queja más frecuente fue la presencia de herida o lesión en la boca $(61,1 \%)$ y la ubicación más común fue la lengua $(62,1 \%)$. Las variables clínicas revelaron estadificación clínica avanzada (III o IV) en 76,4\% de los pacientes. Entre los pacientes de hasta 50 años, hubo una mayor concentración de hombres $(\mathrm{p}=0,015)$ y un mayor consumo de alcohol que entre los pacientes mayores de 50 años $(\mathrm{p}=0,010)$. Las otras variables no mostraron una diferencia estadísticamente significativa entre los grupos. Conclusión: Las características clínicas y epidemiológicas relacionadas con el CCE oral deben considerarse al planificar las políticas públicas, a fin de prevenir nuevos casos y permitir un diagnóstico precoz. Palabras clave: carcinoma de células escamosas de cabeza y cuello/ epidemiología; neoplasias de la boca; registros médicos.

\footnotetext{
1,2,3,7,8Universidade Estadual do Piauí (Uespi), Centro de Ciências da Saúde. Teresina (PI), Brasil. E-mails: julio080994@gmail.com; cadumourac10@gmail.com; rafaelearcosta@gmail.com; lucianatolstenko@ccs.uespi.br; lucielmasalmito@ccs.uespi.br. Orcid iD: https://orcid.org/0000-0002-5674-9980; Orcid iD: https:// orcid.org/0000-0002-6819-7415; Orcid iD: https://orcid.org/0000-0002-0798-890X; Orcid iD: https://orcid.org/0000-0003-2547-7545; Orcid iD: https://orcid. org/0000-0002-8766-8593

${ }^{4}$ Secretaria de Saúde do Estado do Piauí, Hospital Getúlio Vargas. Teresina (PI), Brasil. E-mail: eduarduuh@hotmail.com. Orcid iD: https://orcid.org/00000002-8312-9373

${ }^{5}$ Hospital São Marcos, Serviço de Odontologia. Teresina (PI), Brasil. E-mail: analuisabuco@gmail.com. Orcid iD: https://orcid.org/0000-0002-2324-0475 ${ }^{6}$ Centro Universitário Uninovafapi. Teresina (PI), Brasil. E-mail: pesquisa@amostragem.com.br. Orcid iD: https://orcid.org/0000-0002-1836-5185 Endereço para correspondência: Rafael Everton Assunção Ribeiro da Costa. Rua Olavo Bilac, 2335 - Centro. Teresina (PI), Brasil. CEP 64001-280. E-mail: rafaelearcosta@gmail.com
} 


\section{INTRODUÇÃO}

Segundo estimativas do Instituto Nacional de Câncer José Alencar Gomes da Silva (INCA), são previstos 625 mil casos novos de câncer no Brasil para cada ano do triênio 2020-2022, dos quais, são esperados 15.210 apenas de câncer bucal (11.200 em homens e $4.010 \mathrm{em}$ mulheres), figurando como o quinto mais frequente no sexo masculino e o $13^{\circ}$ mais frequente no sexo feminino, excetuando-se o câncer de pele náo melanoma ${ }^{1}$. Os cânceres da cavidade oral, isto é, os que ocorrem em lábios, mucosa bucal, parte anterior da língua, assoalho da boca, palato duro e gengiva superior e inferior, correspondem a cerca de $30 \%$ de todos os tumores de cabeça e pescoço ${ }^{2}$.

Aproximadamente $90 \%$ dos tumores malignos que ocorrem na cavidade oral são carcinomas espinocelulares (CEC), enquanto os $10 \%$ restantes representam malignidades raras (variantes do carcinoma de células escamosas, melanomas, linfomas e sarcomas) e uma variedade de tumores malignos de origem odontogênica ${ }^{3,4}$.

O CEC oral deve ser buscado por meio do exame clínico da mucosa oral em consultas de rotina em toda a população, porém especialmente em indivíduos que apresentam exposição a um ou mais fatores de risco, como o consumo de álcool e tabaco ${ }^{3}$. Um aumento na incidência de CEC oral tem sido apontado entre indivíduos jovens, com idade até 50 anos e, em mulheres, apesar de o perfil clássico dos pacientes afetados ainda ser de homens na sexta década de vida ${ }^{4,5}$.

A falta de informação da população geral e dos profissionais de saúde sobre os fatores associados a essa neoplasia atrasa o diagnóstico, o que diminui a eficácia do tratamento e aumenta a gravidade das sequelas secundárias a ele, principalmente relacionadas à deglutição e à fonação, além de elevar os índices de mortalidade ${ }^{3,4}$.

Poucos estudos têm avaliado as características epidemiológicas e clinicopatológicas do CEC oral no Brasil, sendo que a maioria foi conduzida na Região Sudeste do país, refletindo a escassez de informaçóes relativas a esse tipo de neoplasia no Nordeste brasileiro ${ }^{5,6}$. Desse modo, o objetivo do presente estudo foi avaliar os aspectos clínico-epidemiológicos de pacientes com CEC oral atendidos em um centro de referência em Oncologia da Regiāo Nordeste do Brasil.

\section{MÉTODO}

Estudo observacional, com delineamento transversal, quantitativo e retrospectivo, a partir da avaliaçáo dos prontuários de pacientes com diagnóstico histopatológico de CEC bucal atendidos em um centro de referência em Oncologia localizado em Teresina (PI), Brasil. A coleta de dados ocorreu entre janeiro e maio de 2019. Foram incluídos no estudo pacientes de ambos os sexos e de qualquer idade cujo atendimento inicial tivesse ocorrido em janeiro de 2014 ou em datas anteriores, pois assim seria possível observar as variáveis clínicas com pelo menos cinco anos de seguimento. Foram excluídos todos os pacientes cujos prontuários não estivessem adequadamente preenchidos.

As variáveis demográficas (sexo, idade, procedência e raça), comportamentais (hábitos tabagista e etilista) e clínicas (queixas no primeiro atendimento, local da lesão, estadiamento clínico e tratamento) foram coletadas dos prontuários. Consideraram-se para este estudo as seguintes regiôes anatômicas: língua, assoalho, palato, lábio, área retromolar, vestíbulo da boca e outros (mucosa jugal, amígdala e gengivas), conforme a $10^{a}$ Edição da Classificação Internacional de Doenças e Problemas Relacionados à Saúde $(\mathrm{CID}-10)^{7}$. Para correlação das variáveis entre os grupos, pacientes com a idade até 50 anos foram considerados jovens; e pacientes acima de 50 anos foram considerados idosos.

Os dados foram tabulados e analisados no programa Statistical Package for the Social Sciences ${ }^{\circledR}$ (SPSS Statistic Data) versão 25.0. Aplicou-se o teste qui-quadrado de Pearson para avaliar a diferença nas proporçóes de desfechos categóricos, sendo considerado um nível de significância de $5 \%$.

O presente estudo foi realizado após aprovação pelo Comitê de Ética em Pesquisa (CEP), sob o número CAAE: 87297218.6.3001.5584, sendo respeitadas todas as diretrizes éticas em pesquisa vigentes no Brasil, de acordo com a Resolução do Conselho Nacional de Saúde $\mathrm{n}^{\circ} .466 / 12^{8}$.

\section{RESULTADOS}

Foram avaliados 393 pacientes com diagnóstico histopatológico de CEC bucal. Excluíram-se 95 prontuários por causa da ausência de dados ou do preenchimento incompleto, sendo incluídos ao final 298 pacientes no estudo. Os pacientes apresentaram idade média de 60,4 anos, e foram mais afetados os indivíduos do sexo masculino $(75,2 \%)$, com faixa etária entre 50 e 60 anos (26,5\%), pardos ou negros $(85,9 \%)$ e oriundos do interior do Estado (56,4\%). A queixa mais reportada foi a presença de ferida ou lesão na boca $(61,1 \%)$. Tabaco e álcool foram os fatores comportamentais mais apresentados, ocorrendo simultaneamente em $62,0 \%$ dos pacientes. Os sítios anatômicos mais prevalentes foram a língua $(62,1 \%)$ e o assoalho oral $(12,1 \%)$.

As variáveis clínicas revelaram tamanho tumoral avançado T4 em 53,6\% dos pacientes, ausência de 
comprometimento linfonodal cervical em $56,7 \%$ e ausência de metástases a distância em $89,2 \%$, resultando em estadiamento TNM IV em $62,7 \%$ dos casos ${ }^{9}$. A conduta terapêutica mais utilizada foi a combinaçáo entre radioterapia e quimioterapia $(53,0 \%)$ (Tabela 1 ). $\mathrm{O}$ seguimento médio (intervalo de tempo entre a data do diagnóstico histopatológico e a última consulta) desses pacientes foi de 17,7 meses.

Tabela 1. Características epidemiológicas e clínicas de 298 pacientes com diagnóstico de CEC bucal

\begin{tabular}{|c|c|c|}
\hline Variáveis & $\mathbf{N}$ & $\%$ \\
\hline \multicolumn{3}{|l|}{ Sexo } \\
\hline Masculino & 224 & 75,2 \\
\hline Feminino & 74 & 24,8 \\
\hline \multicolumn{3}{|l|}{ Faixa etária } \\
\hline$\leq 40$ anos & 19 & 6,3 \\
\hline $41-50$ anos & 57 & 19,1 \\
\hline $51-60$ anos & 79 & 26,5 \\
\hline $61-70$ anos & 67 & 22,4 \\
\hline $71-80$ anos & 52 & 17,4 \\
\hline$>80$ anos & 24 & 8,3 \\
\hline \multicolumn{3}{|l|}{ Cor da pele } \\
\hline Pardos/negros & 256 & 85,9 \\
\hline Brancos & 34 & 11,4 \\
\hline Sem informação & 8 & 2,7 \\
\hline \multicolumn{3}{|l|}{ Procedência } \\
\hline Capital & 88 & 29,5 \\
\hline Interior do Estado & 168 & 56,4 \\
\hline Outros Estados & 42 & 14,1 \\
\hline \multicolumn{3}{|l|}{ Queixa principal } \\
\hline Ferida na boca & 182 & 61,1 \\
\hline Dor na boca & 26 & 8,7 \\
\hline Odinofagia & 31 & 10,4 \\
\hline Nódulo no pescoço & 29 & 9,7 \\
\hline Disfagia & 16 & 5,4 \\
\hline Outros & 14 & 4,7 \\
\hline \multicolumn{3}{|c|}{ Fatores comportamentais associados } \\
\hline Tabagista e etilista & 185 & 62,0 \\
\hline Apenas tabagista & 82 & 27,5 \\
\hline Apenas etilista & 8 & 2,7 \\
\hline Sem hábitos & 17 & 5,8 \\
\hline Não informado & 6 & 2,0 \\
\hline
\end{tabular}

continua
Tabela 1. continuação

\begin{tabular}{lcc}
\hline Variáveis & N & $\%$ \\
\hline Local da lesão & & \\
Língua & 185 & 62,1 \\
Assoalho da boca & 36 & 12,1 \\
Palato & 22 & 7,4 \\
Lábio & 15 & 5,0 \\
Área retromolar & 13 & 4,4 \\
Vestíbulo da boca & 11 & 3,7 \\
Outros & 16 & 5,2 \\
Tamanho do tumor (T) & & \\
T1 & 30 & 10,0 \\
T2 & 51 & 17,5 \\
T3 & 51 & 17,5 \\
T4 & 159 & 53,6 \\
Tx & 5 & 1,6 \\
Acometimento linfonodal (N) & & \\
N0 & 169 & 56,7 \\
N1 & 44 & 14,7 \\
N2 & 46 & 15,4 \\
N3 & 39 & 13,2 \\
Metástase a distância (M) & & \\
M0 & 266 & 89,2 \\
M1 & 12 & 4,0 \\
Estadiamento clínico (TNM) & & \\
I & 37 & 12,4 \\
II & 10 & 31 \\
IV & 31 & 10,4 \\
Não informado & 41 & 13,7 \\
Cratamento & 187 & 62,7 \\
Radioterapia & 2 & 0,6 \\
Quimioterapia & & \\
Cirurgia + radioterapia & 10 & 3,3 \\
Radioterapia + quimioterapia & 17 & 5,7 \\
\hline
\end{tabular}

Comparando-se as variáveis sociodemográficas e clínicas de acordo com as faixas etárias, observou-se que, entre os pacientes jovens, houve uma maior concentração de homens ( $\mathrm{p}=0,015)$ e maior consumo de álcool do que entre os pacientes acima dos 50 anos $(\mathrm{p}=0,010)$. As demais variáveis não exibiram diferença estatística significante entre os grupos (Tabela 2). 
Tabela 2. Comparação entre as variáveis estudadas de acordo com as faixas etárias até 50 anos e a partir de 50 anos de 298 pacientes com diagnóstico de CEC bucal

\begin{tabular}{|c|c|c|c|c|c|c|c|}
\hline \multirow{3}{*}{ Variáveis } & \multicolumn{4}{|c|}{ Idade ao diagnóstico (anos) } & \multirow{3}{*}{ Média } & \multirow{3}{*}{ DP } & \multirow{3}{*}{$\mathbf{p}$} \\
\hline & \multicolumn{2}{|c|}{$\leq \mathbf{5 0}$} & \multicolumn{2}{|c|}{$>\mathbf{5 0}$} & & & \\
\hline & $\mathbf{N}$ & $\%$ & $\mathbf{N}$ & $\%$ & & & \\
\hline \multicolumn{8}{|l|}{ Sexo* } \\
\hline Masculino & 65 & 85,5 & 159 & 71,6 & 59 & 13 & \multirow{2}{*}{0,015} \\
\hline Feminino & 11 & 14,5 & 63 & 28,4 & 65 & 13 & \\
\hline \multicolumn{8}{|l|}{ Procedência } \\
\hline Capital & 23 & 30,3 & 65 & 29,3 & 60 & 14 & \multirow{2}{*}{0,871} \\
\hline Interior/outros Estados & 53 & 69,7 & 157 & 70,7 & 60 & 14 & \\
\hline \multicolumn{8}{|l|}{ Queixa principal } \\
\hline Ferida na boca & 48 & 63,2 & 134 & 60,4 & 60 & 14 & \multirow{2}{*}{0,666} \\
\hline Outras queixas & 28 & 36,8 & 88 & 39,6 & 60 & 13 & \\
\hline \multicolumn{8}{|l|}{ Tabagismo $^{a}$} \\
\hline Sim & 66 & 89,2 & 201 & 92,2 & 60 & 14 & \multirow{2}{*}{0,424} \\
\hline Não & 8 & 10,8 & 17 & 7,8 & 61 & 15 & \\
\hline \multicolumn{8}{|l|}{ Etilismo ${ }^{a *}$} \\
\hline Sim & 58 & 78,4 & 135 & 61,9 & 58 & 14 & \multirow{2}{*}{0,010} \\
\hline Não & 16 & 21,6 & 83 & 38,1 & 64 & 13 & \\
\hline \multicolumn{8}{|l|}{ Sítio (local da lesão) } \\
\hline Língua & 53 & 69,7 & 132 & 59,5 & 58 & 13 & \multirow{2}{*}{0,111} \\
\hline Outros locais & 23 & 30,3 & 90 & 40,5 & 64 & 15 & \\
\hline \multicolumn{8}{|l|}{ Tamanho do tumor $(\mathrm{T})^{a}$} \\
\hline $\mathrm{T} 1$ e T2 & 20 & 26,7 & 61 & 28,2 & 62 & 14 & \multirow{2}{*}{0,793} \\
\hline $\mathrm{T} 3$ e T4 & 55 & 73,3 & 155 & 71,8 & 60 & 13 & \\
\hline \multicolumn{8}{|l|}{ Acometimento linfonodal ( $N$ ) } \\
\hline NO & 39 & 51,3 & 130 & 58,6 & 62 & 14 & \multirow{2}{*}{0,271} \\
\hline $\mathrm{N} 1 / \mathrm{N} 2 / \mathrm{N} 3$ & 37 & 48,7 & 92 & 41,4 & 58 & 13 & \\
\hline \multicolumn{8}{|l|}{ Estadiamento clínico $\left(\right.$ TNM) ${ }^{a}$} \\
\hline I e II & 16 & 21,3 & 52 & 23,5 & 63 & 14 & \multirow{2}{*}{0,606} \\
\hline III e IV & 59 & 78,7 & 169 & 76,5 & 60 & 13 & \\
\hline \multicolumn{8}{|l|}{ Tratamento cirúrgico } \\
\hline Sim & 21 & 27,6 & 62 & 27,9 & 58 & 13 & \multirow{2}{*}{0,960} \\
\hline Não & 55 & 72,4 & 160 & 72,1 & 61 & 14 & \\
\hline
\end{tabular}

Legenda: DP = Desvio-padrāo.

$\left(^{*}\right)$ Diferença estatisticamente significante ( $\mathrm{p}<0,05$; qui-quadrado de Pearson).

(a) Variável com dados não informados.

\section{DISCUSSÃO}

Foi descrito um aumento da sobrevida global de cerca de $12 \%$ nas últimas duas décadas entre pacientes com CEC oral tratados em centros oncológicos mundialmente, o que se atribui aos avanços recentes em exames de imagem e terapias mais eficazes ${ }^{10}$. Apesar de tal avanço, a sobrevida global em cinco anos, mesmo com ampla variaçáo nas literaturas mundial e nacional, ainda está em torno de
$50 \%{ }^{11}$. Isso se deve em grande parte ao fato de que a maioria dos casos está em estágios avançados no momento da detecção, mesmo com a fácil acessibilidade da cavidade oral para exames regulares ${ }^{3}$, o que deixa evidente a falta de esclarecimento sobre a doença por parte dos pacientes e até mesmo dos profissionais ${ }^{12}$.

Neste estudo, indivíduos do sexo masculino na sexta década de vida foram a maioria dos pacientes afetados, o que está de acordo com a literatura ${ }^{3,4,6,11,13-15}$. A maior prevalência 
em homens está relacionada à maior exposição aos fatores de risco. Adicionalmente, com o avanço da idade, aumenta-se o risco de desenvolver o câncer bucal, com a maioria dos casos ocorrendo acima dos 50 anos. No entanto, tem sido relatada uma tendência de crescimento dos casos de CEC oral entre pacientes mais jovens, bem como entre mulheres, muito provavelmente em decorrência de mudanças no estilo de vida nos últimos anos ${ }^{3,4}$.

Quanto à procedência, a maioria dos pacientes era do interior do Piauí (56,4\%), o que se deve essencialmente à ausência de serviços especializados no interior e à necessidade de busca por centros de referência nas capitais. Com relação à variável raça/cor da pele, houve predomínio de indivíduos autodeclarados pardos ou negros $(85,9 \%)$, fato relacionado também ao predomínio da cor parda e preta na população do Piauí (73\% do total) ${ }^{16}$. Semelhante fato ocorreu em estudo realizado na Bahia, em que aproximadamente $90 \%$ dos pacientes eram pardos ou negros, em conformidade com as etnias predominantes nesse Estado ${ }^{15}$.

No presente estudo, observou-se que $89,5 \%$ e $64,7 \%$ dos pacientes apresentavam hábitos tabagistas e etilistas, respectivamente. Em 62,0\% dos pacientes, ambos os hábitos eram adotados simultaneamente. O fumo e o álcool são os fatores de maior potencial carcinogênico, apesar do aspecto multifatorial da doença ${ }^{3,4,11,12}$. A associação entre tabagismo e CEC bucal já está bem estabelecida. Quando o tabagismo está associado à ingestão crônica de bebidas alcoólicas, observa-se efeito sinérgico dose-dependente ${ }^{14}$. A duração, a frequência e o modo de uso dessas substâncias, assim como sua associação, são diretamente relacionados a um maior risco de CEC oral ${ }^{4}$. Estima-se que o abandono do tabagismo contribui para a redução de 50\% do risco de câncer oral em cinco anos, sendo um importante aspecto para reduzir o câncer bucal ${ }^{3,4}$.

Em relação à região anatômica, prevaleceram os cânceres de língua (62,1\%). Em concordância com outros estudos, a língua confirma-se como o sítio intraoral mais propenso a câncer na maioria das populaçóes ${ }^{3,4,11}$. A maioria dos pacientes tinha como queixa na primeira consulta a presença de lesáo na boca $(61,1 \%)$. Sabe-se que o câncer bucal normalmente se inicia como uma úlcera crônica solitária; entretanto, outras formas podem ser descritas, como placas esbranquiçadas (leucoplasias) ou avermelhadas (eritroplasias), lesōes endofíticas, exofíticas ou ainda verrucosas $3,4,6,12$. É importante que os clínicos suspeitem de lesões bucais solitárias, presentes por tempo superior a três semanas, especialmente se endurecidas, acompanhadas por linfadenopatia cervical ou vistas em pacientes expostos a fatores de risco ${ }^{4}$.

A alta prevalência do diagnóstico tardio foi encontrada neste estudo, visto que o tamanho tumoral correspondeu a
T4 em mais de 50\% dos casos e já havia metástase regional em mais de $40 \%$ dos pacientes. Mais de três quartos dos pacientes se encontravam nos estádios III ou IV da doença. $\mathrm{Na}$ literatura consultada, esse percentual variou de 58,3\% a $82,9 \% 0^{8,14,15,17-20}$.

Com relação às terapêuticas empregadas, a maioria dos indivíduos recebeu como tratamento a combinação de radioterapia e quimioterapia. $\mathrm{O}$ tratamento de escolha para o CEC oral costuma ser a ressecção cirúrgica do tumor primário e, quando necessário, do pescoço, a fim de abordar metástases regionais ${ }^{4,21,22}$. Entretanto, em estádios avançados, como observado no presente estudo, a radioterapia com ou sem quimioterapia é normalmente a terapêutica indicada após a consideração de vários fatores, incluindo idade e comorbidades do paciente ${ }^{22}$.

Um estudo prévio observou que, entre os pacientes com até 50 anos, houve maior concentração de homens e maior número de etilistas do que entre os pacientes mais idosos. A etiologia e os mecanismos pelos quais pacientes jovens desenvolvem CEC oral são virtualmente os mesmos responsáveis pela doença em indivíduos mais velhos, sendo o álcool tradicionalmente considerado um fator de risco importante ${ }^{3}$. Maior presença de homens e etilistas entre os indivíduos jovens com CEC oral pode ser em parte justificada pela maior prevalência do consumo abusivo de álcool na população masculina brasileira ${ }^{23}$.

Não foram observadas diferenças estatisticamente significantes entre as variáveis local ou tamanho da lesão, comprometimento linfonodal, estadiamento clínico geral ou tratamento dos pacientes em jovens e idosos. Estudos prévios têm apresentado resultados inconsistentes e controversos a respeito das diferenças clinicopatológicas e de prognóstico quando comparados pacientes jovens ou idosos com CEC bucal ${ }^{24,25}$. Há estudos indicando que pacientes mais jovens teriam um maior risco de morte causado por maior risco de recorrência ou mestástases ${ }^{24}$, ao passo que outros estudos apontam exatamente o contrário, maior sobrevida entre jovens ${ }^{25}$.

\section{CONCLUSÃO}

O presente estudo evidenciou maior prevalência de CEC oral em homens na sexta década de vida, fumantes e etilistas, com lesão em língua e estadiamento clínico geral avançado (III ou IV) no momento do diagnóstico. Maior concentração de homens e consumo de álcool foram observados entre os pacientes com até 50 anos, o que foi estatisticamente significante. As características clínico-epidemiológicas relacionadas ao CEC bucal devem ser consideradas para o planejamento de políticas públicas, a fim de prevenir novos casos e permitir a realização de diagnóstico precoce. 


\section{CONTRIBUIÇÕES}

Todos os autores contribuíram substancialmente na concepção e/ou planejamento do estudo; na obtenção, análise e/ou interpretaçáo dos dados; na redação e revisão crítica; e aprovaram a versão final a ser publicada.

\section{AGRADECIMENTOS}

À UESPI pelo apoio institucional.

\section{DECLARAÇÃO DE CONFLITO DE INTERESSES}

Nada a declarar.

\section{FONTES DE FINANCIAMENTO}

Não há.

\section{REFERÊNCIAS}

1. Instituto Nacional de Câncer José Alencar Gomes da Silva (INCA). Estimativa 2020: incidência de câncer no Brasil. Rio de Janeiro: INCA; 2019.

2. Campana IG, Goiato MC. Tumores de cabeça e pescoço: epidemiologia, fatores de risco, diagnóstico e tratamento. Rev Odontol Arac. 2013;34(1):20-31.

3. Warnakulasuriya S. Global epidemiology of oral and oropharyngeal cancer. Oral Oncol. 2009;45(4-5):309-16. doi: https://doi.org/10.1016/j.oraloncology.2008.06.002

4. Scully C, Porter S. Oral cancer. West J Med. 2001;174(5):348-51. doi: https://doi.org/10.1136/ ewjm.174.5.348

5. Ribeiro KRB, Lira Júnior $C$, Marinho $S A$, et al. Epidemiological profile of young patients with squamous cell carcinoma in northeast Brazil. J Investig Clin Dent. 2019;10(4):e12436. doi: https://doi.org/10.1111/ jicd. 12436

6. Leite AA, Leonel ACLS, Castro JFL, et al. Oral squamous cell carcinoma: a clinicopathological study on 194 cases in northeastern Brazil. A cross-sectional retrospective study. São Paulo Med J. 2018;136(2):165-9. doi: https:// doi.org/10.1590/1516-3180.2017.0293061217

7. Organização Mundial da Saúde. CID-10: classificação estatística internacional de doenças e problemas relacionados à saúde. 10. ed. São Paulo: Edusp; 2017.

8. Conselho Nacional de Saúde (BR). Resolução no 466, de 12 de dezembro de 2012. Aprova as diretrizes e normas regulamentadoras de pesquisas envolvendo seres humanos. Diário Oficial da União, Brasília, DF. 2013 jun 13; Seção 1:59.

9. União Internacional Contra o Câncer. TNM: classificação de tumores malignos. 7. ed. Rio de Janeiro: INCA; 2012.
10. Amit M, Yen TC, Liao CT, et al. Improvement in survival of patients with oral cavity squamous cell carcinoma: an international collaborative study. Cancer. 2013;119(24):4242-8. doi: https://doi.org/10.1002/ cncr.28357

11. Kowalski LP, Oliveira MM, Lopez RVM, et al. Survival trends of patients with oral and oropharyngeal cancer treated at a cancer center in São Paulo, Brazil. Clinics. 2020;75:e1507. doi: https://doi.org/10.6061/ clinics/2020/e1507

12. Soares TRC, Carvalho MEA, Pinto LSS, et al. Oral cancer knowledge and awareness among dental students. Braz J Oral Sci. 2014;13(1):28-33. doi: https://doi. org/10.1590/1677-3225v13n1a06

13. Moyses RA, López RVM, Cury PM, et al. Significant differences in demographic, clinical, and pathological features in relation to smoking and alcohol consumption among 1,633 head and neck cancer patients. Clinics. 2013;68(6):738-44. doi: https://doi.org/10.6061/ clinics/2013(06)03

14. Daher GCA, Pereira GA, Oliveira ACD. Características epidemiológicas de casos de câncer de boca registrados em hospital de Uberaba no período 1999-2003: um alerta para a necessidade de diagnóstico precoce. Rev Bras Epidemiol. 2008;11(4):584-96. doi: https://doi. org/10.1590/S1415-790X2008000400007

15. Santos LPS, Carvalho FS, Carvalho CAP, et al. Características de casos de câncer bucal no estado da Bahia, 1999-2012: um estudo de base hospitalar. Rev Bras Cancerol. 2015;61(1):7-14. doi: https://doi. org/10.32635/2176-9745.RBC.2015v61n1.350

16. SIDRA: sistema IBGE de recuperação automática [Internet]. Rio de Janeiro: IBGE; [2011] - . [Tabela], Censo 2010: população residente, por cor ou raça, segundo a situação de domicílio, o sexo e a idade; [acesso $2021 \mathrm{fev}$ 11]. Disponível em: https://sidra.ibge.gov.br/tabela/3175

17. Le Campion ACOV, Ribeiro CMB, Luiz RR, et al. Low survival rates of oral and oropharyngeal squamous cell carcinoma. Int J Dent. 2017;2017:5815493. doi: https:// doi.org/10.1155/2017/5815493

18. Santos LCO, Batista OM, Cangussu MCT. Characterization of oral cancer diagnostic delay in the state of Alagoas. Braz J Otorhinolaryngol. 2010;76(4):416-22. doi: https://doi.org/10.1590/ S1808-86942010000400002

19. Abrahão R, Perdomo S, Pinto LFR, et al. Predictors of survival after head and neck squamous cell carcinoma in South America: the InterCHANGE study. JCO Glob Oncol. 2020;6:486-99. doi: https://doi.org/10.1200/ GO.20.00014

20. Kowalski LP, Carvalho AL. Influence of time delay and clinical upstaging in the prognosis of head and neck cancer. Oral Oncol. 2001;37(1):94-8. doi: https://doi. org/10.1016/s1368-8375(00)00066-x 
21. Melo GM, Ribeiro KC, Kowalski LP, et al. Risk factors for postoperative complications in oral cancer and their prognostic implications. Arch Otolaryngol Head Neck Surg. 2001;127(7):828-33.

22. Zanoni DK, Montero PH, Migliacci JC, et al. Survival outcomes after treatment of cancer of the oral cavity (1985-2015). Oral Oncol. 2019;90:115-21. doi: https:// doi.org/10.1016/j.oraloncology.2019.02.001

23. Garcia LP, Freitas LRS. Consumo abusivo de álcool no Brasil: resultados da Pesquisa Nacional de Saúde 2013. Epidemiol Serv Saude. 2015;24(2):227-37. doi: https:// doi.org/10.5123/S1679-49742015000200005

24. Zhang YY, Wang DC, Su JZ, et al. Clinicopathological characteristics and outcomes of squamous cell carcinoma of the tongue in different age groups. Head Neck. 2017;39(11):2276-82. doi: https://doi.org/10.1002/ hed. 24898

25. Chen S, Lin Z, Chen J, et al. Older age is a risk factor associated with poor prognosis of patients with squamous cell carcinoma of the oral cavity. Eur Arch Otorhinolaryngol. 2020;277(9):2573-80. doi: https:// doi.org/10.1007/s00405-020-05963-3

Recebido em 4/3/2021

Aprovado em 11/6/2021 